\title{
SUISEI-A Versatile Global Model of Comets with Applications to Small Solar System Bodies
}

\author{
Daniel C. Boice \\ Scientific Studies and Consulting, San Antonio, USA \\ Email: dcboice@yahoo.com
}

How to cite this paper: Boice, D.C. (2017) SUISEI-A Versatile Global Model of Comets with Applications to Small Solar System Bodies. Journal of Applied Mathematics and Physics, 5, 311-320. https://doi.org/10.4236/jamp.2017.52028

Received: January 18, 2017 Accepted: February 12, 2017 Published: February 15, 2017

\begin{abstract}
SUISEI is a suite of computational tools that has been developed over the past three decades and successfully applied to comets; including ComChem, a global, multi-fluid gas dynamics simulation with detailed chemical kinetics of the cometary coma; ComDust, a model of comet dust evolution and interaction with gas; and ComNuc, a 3-D simulation of gas and heat flow within the comet nucleus porous subsurface layers. The combination of these tools have resulted in an improved knowledge of chemical species in the comet's coma and their relationship to native molecules in the nucleus ices by analyzing space- and ground-based observations and in situ measurements from spacecraft missions. A review of SUISEI is presented and applications are made to two cases: chemical recycling of $\mathrm{HCN}$ in comets and the physical conditions of the near-Sun object, 3200 Phaethon.
\end{abstract}

\section{Keywords}

Comets, Coma Chemistry Models, Reactive Dusty Gas Dynamics, 3200

Phaethon

\section{Introduction}

Comets are believed to be the remnants of the swarm of planetesimals from which the planets formed some 4.6 Gy ago. By investigating in detail the physical and chemical properties of comets, we can characterize the conditions and processes of the Solar System's earliest epoch. Comets are also thought to hold clues to the origins of life since they contain large inventories of water and organics, including prebiotic molecules, on Earth-crossing orbits.

Modeling is central to understand the important properties of the cometary environment. We have developed a comet model, SUISEI (Chinese 彗星; pro- 
nounced "suisei" in Japanese kanji), that self-consistently includes the relevant physicochemical processes within a global modeling framework, from the porous subsurface layers of the nucleus to the interaction with the solar wind and formation of the bow shock and plasma tail. Our goal is to gain valuable insights into the intrinsic properties of cometary nuclei so we can better understand observations and in situ measurements. SUISEI includes a multifluid, reactive gas dynamics simulation of the dusty coma (ComChem) with dust entrainment (ComDust) and transition to free molecular flow, a 3-D code (ComNuc) of gas and heat transport in porous subsurface layers in the interior of the nucleus, and a suite of other coupled numerical simulations. We have successfully applied this model to a variety of comets in previous studies as noted below. After briefly describing these codes, we present two applications of SUISEI to illustrate the chemical recycling of $\mathrm{HCN}$ in cometary come and to investigate the thermal environment of the near-Sun object, 3200 Phaethon.

\section{Description of SUISEI}

SUISEI is a versatile suite of numerical simulations to model the global comet environment. It consists of several codes that can be linked depending on the requirements of a particular application as shown in Figure 1. Three of these codes are summarized below.

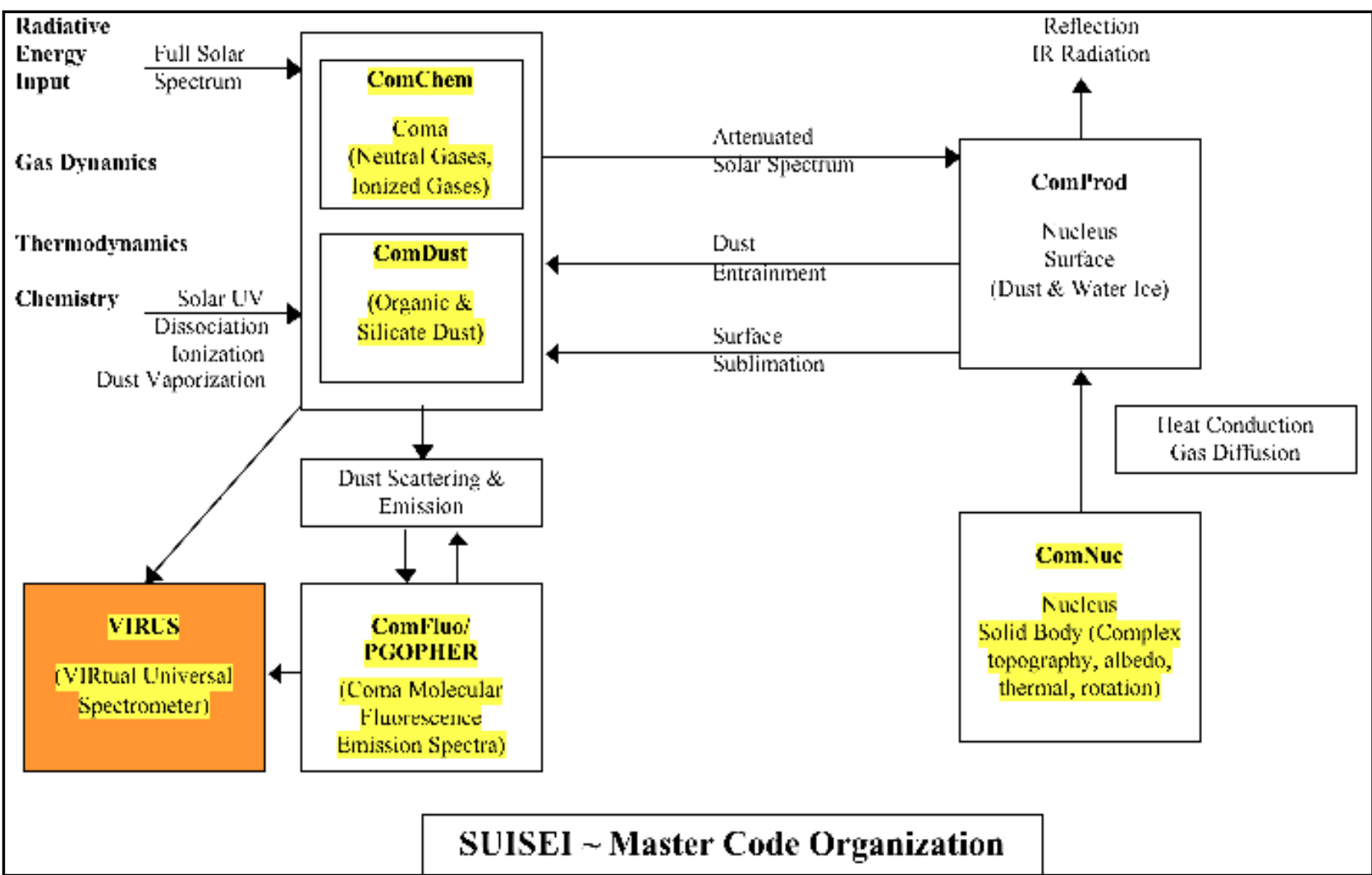

Figure 1. SUISEI Comet Suite illustrating physical inputs and links between the codes. Three sources of coma gas are modeled: sublimation from surface ices, sublimation of supervolatiles from subsurface layers, and release of gas from dust in thecoma, and synthetic molecular emission spectra are produced for comparison with observations. 


\subsection{ComChem-Mulitfluid Gas Dynamics with Detailed Chemistry}

This multifluid gas dynamics model with chemistry is very advanced and has been described by [1] [2] [3] and references therein. ComChem solves the fluid dynamic equationsfor the mass, momentum, and energy of three neutral fluids (atomic and molecular hydrogen and the heavier bulkfluid), ions, and electrons. In the inner coma, the gas expands, cools, accelerates, and undergoes many photolytic and gas-phase chemical reactions tracking hundreds of sibling species. The code handles the transition to free molecular flow and describes the spatial distribution of neutral species in the outer coma of a comet. ComChem produces cometocentric abundances of the coma gas species; velocities of the bulk gas, light atomic and molecular hydrogen with escape, ions, and electrons; gas, ion, and electron temperatures; column densities to aid comparison with observations; coma energy budget; attenuation of the solar irradiance; and other observable quantities.

\subsection{ComDust-Gas-Dust Interaction with Dust Fragmentation}

The ComChem code incorporates our model of dusty gas flow in a cometary atmosphere (ComDust, [4]) that includes gas-drag force (momentum transfer), heat and mass exchange between gas and dust, radiative heating and cooling for dust particles, particle size distribution (dozens of discrete grain sizes), and fragmentation of grains (each grain size with a different lifetime). This code illuminates relevant physical processes for gas-dust interactions, such as distributed gas sources from the dust, mass-loading of gas by the dust, and energy exchange between gas and dust components. Profiles of the number density of dust as a function of size, dust velocities and temperatures as a function of size, and fragmentation rates are produced and related to observations. Model results are integrated in the line of sight for direct comparison with observations. The dust model includes the jet-like nature of the sources and has been applied successfully to comets 1 P/Halley and 19P/Borrelly [5].

\subsection{ComNuc-Gas and Heat Flow in a Porous Body}

We have developed a 3-D simulation of gas and heat flow within the comet nucleus subsurface layers (ComNuc, [6]). This code has been successfully linked with ComChem to study comet Hyakutake [7]. With ComNuc enhanced with detailed chemistry and full dusty gas interaction, unique in the community, comprehensive descriptions and understanding of comets can be achieved, including the processes of devolatilization and mantle formation. We use results from a variety of comet nucleus models for heat and gas diffusion in porous, subsurface layers, including recent laboratory measurements, leveraging years of work in this community [8]. The combined model incorporates gas/dustproduction from three sources: volatile sublimation at the nucleus surface, subsurface sublimation of volatiles from the interior, and release of gas from the dust grains in the coma (distributed sources). From the simulations, we obtain temperature, relative chemical abundance, porosity, pore size distributions as a 
function of depth, and the gas flux into the interior and into the coma for each of the volatiles at various positions of the comet in its orbit.

\section{Applications of SUISEI to Small Solar System Objects}

The solar radiation field initiates most of the processes that occur in cometary comae. Photons at ultraviolet (UV) wavelengths photo dissociate and ionize the original parent molecules, producing second-generation reactive radicals, ions, and electrons. These ions and radicals can subsequently react with other species to form third-generation species. Photoelectrons are an additional source of ionization (and dissociation) via impact reactions [9] [10]. Figure 2 shows SUISEI results of the coma neutral gas temperature from simulations of a comet traversing from 2.5 $\mathrm{AU}$ to $0.3 \mathrm{AU}$ pre-perihelion in its orbit. Large increases in the gas temperature can be seen within about $0.5 \mathrm{AU}$, with dramatic effects on the chemistry, optical depth, and other coma properties.

\subsection{Chemical Recycling of HCN in Cometary Comae}

$\mathrm{CN}$ has been seen in cometary spectra for over a century but its source(s) remains unknown. A CN source must be able to produce highly collimated $\mathrm{CN}$ "jets" be consistent with the observed $\mathrm{CN}$ parent scale length, have a production rate consistent with the observed $\mathrm{CN}$ production, and isotopic ratios of $\mathrm{C}$ and $\mathrm{N}$ must be consistent. HCN fulfills these conditions in some comets [e.g., 1P/Halley, C/2002 T7 (LINEAR), 17P/Holmes, 73P/S-W 3, 2P/Encke] while it does not in others [e.g., C/1983 H1 (IAA), C/1995 O1 (Hale-Bopp), C/2001 Q4, 8P/Tuttle, 6P/d'Arrest] [11]. The chemistry of $\mathrm{HCN}$ was investigated with SUISEI including other possible CN parents, as well as a dust component as a

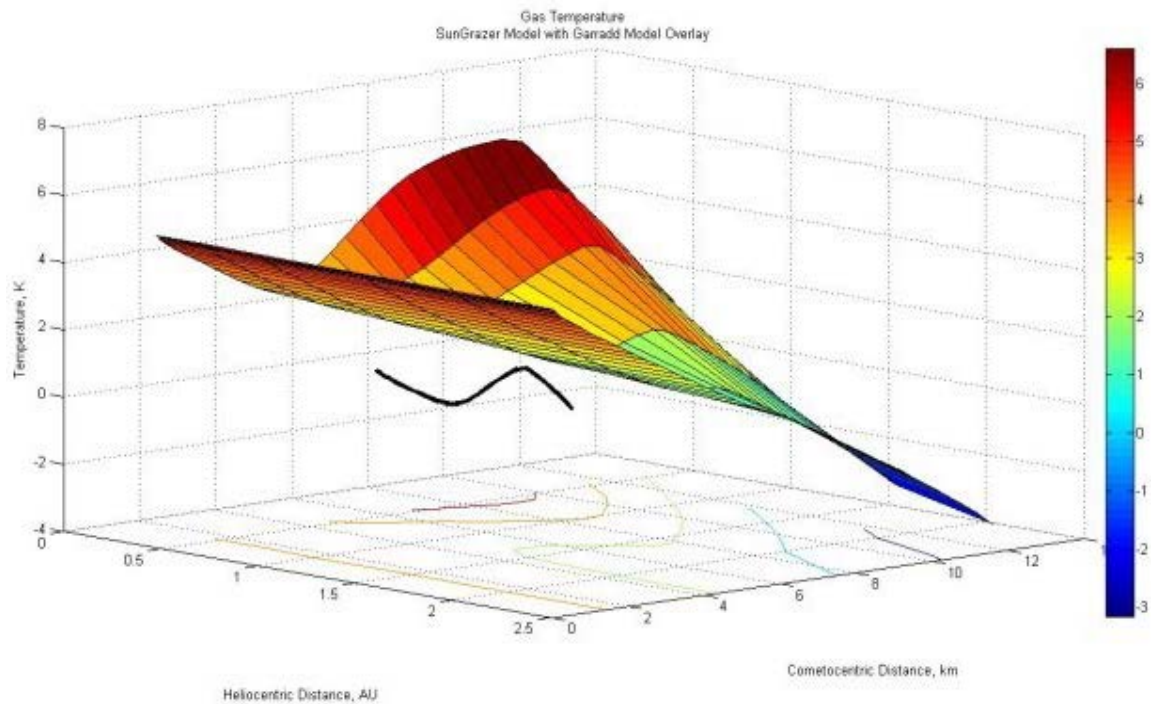

Figure 2. Variation of gas temperature $\left[\ln \left(T_{g}\right)\right.$ in $\left.K\right]$ with cometocentric distance [in $\ln (\mathrm{R}) \mathrm{km}$ ] and heliocentric distance [ $\mathbf{r}$ in AU] for a comet approaching the Sun from 2.5 to $0.3 \mathrm{AU}$. Comet Garradd at 1.6 AU has been added for reference (black line). Note the enhanced gas temperatures $(>400 \mathrm{~K})$ due to photolytic heating in the coma within $\sim 0.5$ AU. 
potential source [12]. It is seen in Figure 3 that the major destruction pathways of $\mathrm{HCN}$ are via photo dissociation (into $\mathrm{H}$ and $\mathrm{CN}$ ) and protonation with water group ions, primarily, $\mathrm{H}_{3} \mathrm{O}^{+}$. We find that $\mathrm{HCN}$ molecules are "recycled" via protonation reactions with $\mathrm{H}_{3} \mathrm{O}^{+}, \mathrm{H}_{2} \mathrm{O}^{+}, \mathrm{OH}^{+}$, and subsequent electron dissociative recombination that leaves the newly formed $\mathrm{HCN}$ molecule in an excited state, leading to prompt emission of "hot" rotational-vibrational bands. In addition, the recombination process converts a fraction of molecules to the isomer, HNC, also observed in comets [13]. HCN molecules observed in the coma can consist of those initially released from the nucleus (native) and those that are freshly formed at different locations in the coma via these protonation/recombination reactions. For a modest production rate comet such as C/2012 F6 (Lemmon), we find that the recycled component is only a few percent of the native component [14]. This isn't sufficient to reconcile discrepancies between IR and radio observations of HCN or HNC abundances in cometary comae. Our study continues for comets with higher production rates where inner coma chemistry is more effective. Chemical recycling will be an important process for additional cometary molecules with proton affinities greater than water, such as, $\mathrm{NH}_{3}$, water clusters, and others (including $\mathrm{H}_{2} \mathrm{O}$ ). We note high-resolution spectral observations of comets show "hot" bands of $\mathrm{NH}_{3}$, water, and others in the inner coma [15].

\subsection{Near-Sun Comets and 3200 Phaethon}

The near-Sun object 3200 Phaethon was discovered in 1983 and classified as an asteroid [16]. It was recognized that its orbit is very similar to that of the Geminid meteoroid stream [17]. Phaethon exhibits a faint but active dust coma [19],

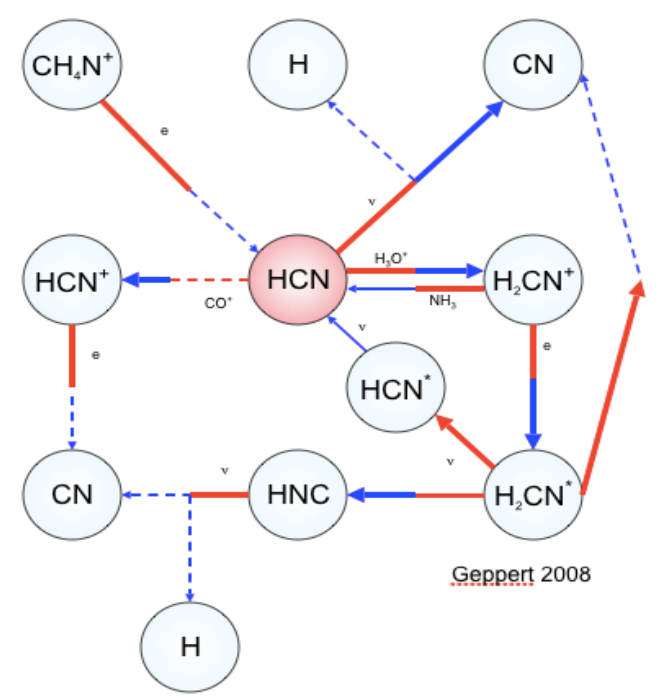

Figure 3. Chemistry of $\mathrm{HCN}$ in the inner coma of a typical comet. Note the recycling loop where $\mathrm{HCN}$ is protonated by $\mathrm{H}_{3} \mathrm{O}^{+}$to form $\mathrm{H}_{2} \mathrm{CN}^{+}$that dissociates via electron recombination, forming an excited $\mathrm{HCN}^{\star}$ molecule that promptly emits rotational-vibrational radiation ("hot bands") to return to the ground state. Some HNC is also formed. [blue arrow $=$ production, red $=$ destruction; thick = major pathway, thin $=$ average, dot ted $=$ minor $]$. 
providing a clear link to the Geminids. The observed dust activity would traditionally lead to Phaethon's classification as a comet, e.g., as in 7968-133P/ ElstPizarro. However, Phaethon's orbit has a perihelion of only 0.14 AU, resulting in subsolar surface temperatures $>1000 \mathrm{~K}$, much too hot for water ice or other volatiles to exist near the surface to drive the activity like a traditional comet. At heliocentric distances $<0.2 \mathrm{AU}$, temperatures are high enough to vaporize surface hydrocarbons and dust, providing a source of gas. This led [18] to suggest thermal or desiccation cracking as the driving mechanism, and to refer to Phaethon as an "active asteroid." This situation (and others such as the "main belt comets" or "active asteroids") necessitates a revision of how we understand and classify these small comet-asteroid transition objects but we will not discuss it further here.

An important question concerns the energy balance at the body's surface, namely what fraction of incident energy will be conducted into the interior versus that expended through sublimation. It is important to understand whether the interior remains cold and is relatively unaltered during each perihelion passage or is significantly devolatilized. Since Phaethon exhibits a mixture of cometary and asteroidal properties, it may be an extinct or dormant comet nucleus. Time-dependent thermal modeling using ComNuc [19] shows in Figure 4 the

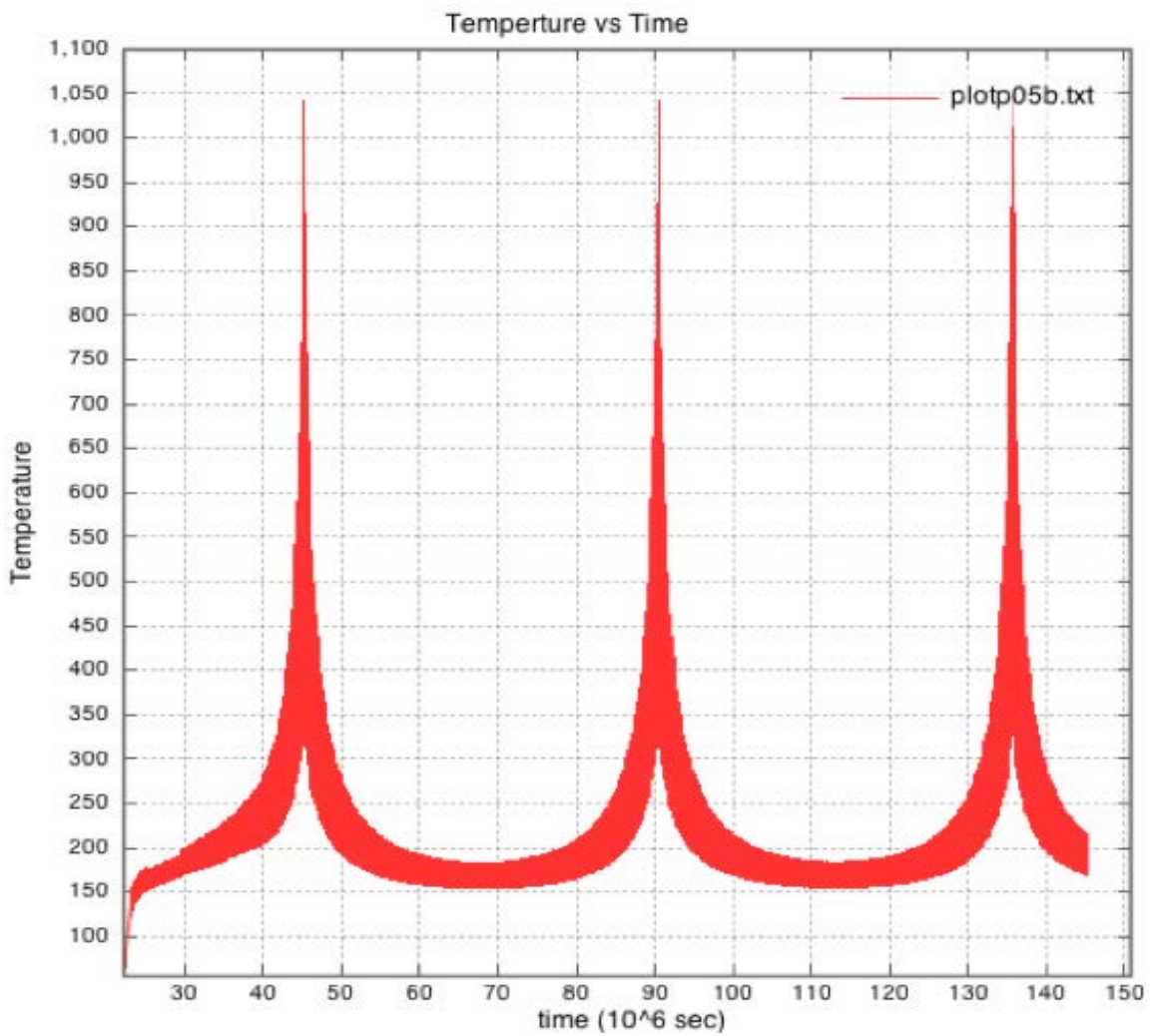

Figure 4. Variation of surface temperature $\left[\mathrm{T}_{\mathrm{s}}\right.$ in $\left.\mathrm{K}\right]$ with time $\left[\mathrm{t}\right.$ in $\left.10^{6} \mathrm{~s}\right]$ along the first 3 orbits of 3200 Phaethon. Note the large diurnal effects that Phaethon experiences during 3.604 hours around perihelion and the extreme changes of temperature during its orbit of 1.43 years. The SUISEI results are consistent with the STM, FRM, and NEATM models (see text). 
temperature evolution along Phaethon's orbit at the subsolar point as it undergoes diurnal rotation (period of 3.604 hours). It is noted that the subsolar temperature is consistent with the standard thermal model, STM [20]. Diurnal variations are extreme at perihelion, resulting in temperature changes in excess of $700 \mathrm{~K}$ in 1.8 hours, and are consistent with the near-Earth asteroid thermal model, NEATM [21] that assumes very low thermal inertia. The average surface temperature, integrated over the entire surface is consistent with the so-called fast-rotating model, FRM [22] that assumes a very high thermal inertia and/or very fast rotation. Similar results for surface temperatures were obtained by [23].

Model results also confirm the likelihood of large thermal stresses in the surface layers of Phaethon, leading to fractures and the release of dust particles as suggested by [18]. Thermal cycling from $1000 \mathrm{~K}$ to $300 \mathrm{~K}$ at perihelion due to rotation most likely leads to the disintegration of surface rocks that form the observed dust coma and material for the meteor stream. Extreme temperature gradients are also expected beneath the surface, especially at perihelion, which will also fuel the dust release.

The gas flux of $\mathrm{H}_{2} \mathrm{O}$ along Phaethon's orbit for two thermal conductivities (one typical of asteroids, the other typical of comets) is sharply peaked around perihelion. After the initial approach, the water gas flux is under $10^{-8} \mathrm{~kg} \cdot \mathrm{m}^{-2} \cdot \mathrm{s}^{-1}$ in all cases. At these low gas fluxes, it would take about $2 \mathrm{My}$ to devolatilize a surface layer of $10 \mathrm{~m}$ depth on Phaethon. The interior of Phaethon may therefore still have a relatively primitive volatile inventory despite repeated close approaches to the Sun. This is due to the low thermal conductivity of the object, making the surface layers an effective thermal insulator. With maximum gas flux at perihelion being a few $10^{-9} \mathrm{~kg} \cdot \mathrm{m}^{-2} \cdot \mathrm{s}^{-1}$, total water production is about $1 \mathrm{~kg} \cdot \mathrm{s}^{-1}$. This is not sufficient to entrain the observed dust production [24] so the possibility of steady water sublimation as the driver of the dust activity is ruled out. However, it is possible that an impulsive gas outburst, triggered by pockets of subsurface gas suddenly bursting through the surface or other mechanisms, may be responsible, similar to the outbursts of comets 17P/Holmes and 1P/Halley seen at larger heliocentric distances.

We conclude that: 1) Phaethon may contain primitive volatiles in its interior despite repeated perihelion passages at $0.14 \mathrm{AU}$ during its history in its present orbit, 2) steady water gas fluxes at perihelion and throughout its orbit are likely insufficient to entrain the currently observed dust production, 3) thermal gradients beneath the surface as well as those caused by rotation are consistent with the mechanism of dust release due to repeated thermal fracture, and 4) the large gas release during the first several perihelion passages may be sufficient to produce enough dust to explain the entire meteoroid stream.

\section{Conclusions}

Using SUISEI, we have conducted simulations of HCN in comets and found that it undergoes ion-molecule chemistry in the inner coma, including protonation with water-group ions followed by electron dissociative recombination that "re- 
cycles" molecules, resulting in its extended range in the coma, formation of $\mathrm{HNC}$, and rotational-vibrational emission bands from highly excited states. Chemical recycling of other molecules with high proton affinities such as, $\mathrm{NH}_{3}$ and $\mathrm{H}_{2} \mathrm{O}$, will occur and possibly provide an explanation for spectral observations showing "hot" bands of these molecules and others in the inner coma.

We have investigated the hypothesis that Phaethon may be a dormant or extinct comet nucleus that is still experiencing low level water gas production as the driver of its recently observed dust activity. The model shows the extreme heat cycling that Phaethon experiences during each perihelion passage and its diurnal variation. Our results indicate that although Phaethon may still harbor a volatile reservoir in its interior despite repeated perihelion passages due to its very low thermal conductivity typical of small solar system bodies. The water gas production rates are not sufficient to explain the observed dust production rates. The possibility that Phaethon is a dormant comet nucleus is not ruled out.

SUISEI has proven to be a unique and valuable model to understand the relevant physical processes and properties of small Solar System bodies, including near-Sun comets [25].

\section{Acknowledgements}

This work was supported by Fundação de Amparo à Pesquisa do Estado de São Paulo (FAPESP, Brazil, Grant No. 2015/03176-8) and the National Science Foundation Planetary Astronomy Program (NSF, USA, Grant No. 0908529).

\section{References}

[1] Giguere, P.T. and Huebner, W.F. (1978) A Model of Comet Comae. I-Gas-Phase Chemistry in One Dimension. Astrophys. J., 223, 638-654. https://doi.org/10.1086/156298

[2] Rodgers, S.D., Charnley, S.B., Huebner, W.F. and Boice, D.C. (2004) Physical Processes and Chemical Reactions in Cometary Comae. In: Festou, M.C., Keller, H.U. and Weaver, H.A., Eds., Comets II, Univ. of Arizona Press, Tucson, 505-522.

[3] Boice, D.C. and Wegmann, R. (2007) The Deep Space 1 Encounter with Comet 19P/Borrelly. Adv. Space Res., 39, 407-412. https://doi.org/10.1016/j.asr.2003.02.092

[4] Konno, I., Huebner, W.F. and Boice, D.C. (1993) A Model of Dust Fragmentation in Near-Nucleus Jet-Like Features on Comet P/Halley. Icarus, 101, 84-94. https://doi.org/10.1006/icar.1993.1008

[5] Boice, D.C., Soderblom, L.A., Britt, D.T., Brown, R.H., Sandel, B.R., Yelle, R.V., Buratti, B.J., Hicks, M.D., Nelson, R.M., Rayman, M.D., Oberst, J. and Thomas, N. (2002) The Deep Space 1 Encounter with Comet 19P/Borrelly. Earth, Moon, Planets, 89, 301-324. https://doi.org/10.1023/A:1021519124588

[6] Benkhoff, J. and Huebner, W.F. (1995) Influence of the Vapor Flux on Temperature, Density, and Abundance Distributions in a Multicomponent, Porous, Icy Body. Icarus, 114, 348-354. https://doi.org/10.1006/icar.1995.1067

[7] Benkhoff, J. and Boice, D.C. (1996) Modeling the Thermal Properties and the Gas Flux from a Porous, Ice-Dust Body in the Orbit of P/Wirtanen. Planet. Space Sci., 44, 665-673. https://doi.org/10.1016/0032-0633(96)00047-5 
[8] Huebner, W.F., Benkhoff, J., Capria, M.-T., Coradini, A., De Sanctis, C., Orosei, R. and Prialnik, D. (2004) Heat and Gas Diffusion in Comet Nuclei. ESA Publications Division, Noordwijk, The Netherlands.

[9] Ip, W.-H. (1985) A Preliminary Consideration of the Electron Impact Ionization Effect in Cometary Comas. Adv. Space Res., 5, 47-51. https://doi.org/10.1016/0273-1177(85)90066-3

[10] Boice, D.C., Huebner, W.F., Keady, J.J., Schmidt, H.U. and Wegmann, R. (1986) A Model of Comet P/Giacobini-Zinner. Geophys. Res. Lett., 13, 381-384. https://doi.org/10.1029/GL013i004p00381

[11] Fray, N., Bénilan, Y., Cottin, H., Gazeau, M.-C. and Crovisier, J. (2005) The Origin of the CN Radial in Comets: A Review from Observations and Models. Planet. Space Sci., 53, 1243-1262. https://doi.org/10.1016/j.pss.2005.06.005

[12] Boice, D.C., Kawakita, H., Shinnaka, Y. and Kobayashi, H. (2015) Chemical Recycling in the Comae of Comets. Proceedings of the 46th Lunar and Planetary Science Conference, The Woodlands, Texas, 16-20 March 2015, LPI Contribution No. 1832, 1749.

[13] Geppert, W.D. and Larsson, M. (2008) Dissociative Recombination in the Interstellar Medium and Planetary Ionospheres. Molecular Physics, 106, 2199-2226. https://doi.org/10.1080/00268970802322074

[14] Boice, D.C., Kawakita, H., Shinnaka, Y., Mumma, M.J., Kobayashi, H. and Ogawa, S. (2014) Chemical Recycling of HCN in Cometary Comae. Proceedings of the American Astronomical Society, Division of Planetary Science Meeting \#46, Tucson, Arizona, 9-14 November 2014, ID: 103.06.

[15] Dello Russo, N., Vervack, R.J. and Kawakita, H. (2014) The Production and Release of Volatiles in Comets. Proceedings of the American Astronomical Society, Division of Planetary Science Meeting \#46, Tucson, Arizona, 9-14 November 2014, ID: 209.12.

[16] Green, S. and Kowal, C. (1983) 1983 TB. IAU Circular, No. 3878, \#1, Marsden, B.G., Ed.

[17] Whipple, F.L. (1983) 1983 TB and the Geminid Meteors. IAU Circular, No. 3881, \#1, Marsden, B.G., Ed.

[18] Jewitt, D. and Li, J. (2010) Activity in Geminid Parent (3200) Phaethon. Astronomical J., 140, 1519-1527. https://doi.org/10.1088/0004-6256/140/5/1519

[19] Boice, D.C. and Benkhoff, J. (2015) Modeling the Near-Sun Object, 3200 Phaethon. Proceedings of the 46th Lunar and Planetary Science Conference, The Woodlands, Texas, 16-20 March 2015, LPI Contribution No. 1832, 1781.

[20] Lebofsky, L.A., Sykes, M.V., Tedesco, E.F., Veeder, G.J., Matson, D.L., Brown, R.H., Gradie, J.C., Feierberg, M.A. and Rudy, R.J. (1986) A Refined 'Standard' Thermal Model for Asteroids Based on Observations of 1 Ceres and 2 Pallas. Icarus, 68, 239251. https://doi.org/10.1016/0019-1035(86)90021-7

[21] Harris, A.W. (1998) A Thermal Model for Near-Earth Asteroids. Icarus, 131, 291301. https://doi.org/10.1006/icar.1997.5865

[22] Lebofsky, L.A. and Spencer, J.R. (1989) Radiometry and Thermal Modeling of Asteroids. In: Asteroids II; Proceedings of the Conference, Tucson, Arizona, 8-11 March 1988, University of Arizona Press, 128-147.

[23] Ohtsuka, K., Nakato, A., Nakamura, T., Kinoshita, D., Ito, T., Toshikawa, M. and Hasegawa, S. (2009) Solar-Radiation Heating Effects on 3200 Phaethon. Publications of the Astronomical Society of Japan, 61, 1375-1387.

https://doi.org/10.1093/pasj/61.6.1375 
[24] Jewitt, D. (2013) Properties of Near-Sun Asteroids. Astronomical J., 145, ID: 133, 6 p.

[25] Jones, G.H., Battams, K., Birkett, K., Boice, D.,C., Brown, J., Bzowski, M., Decock, A., Fitzsimmons, A., Giordano, S., Knight, M. Lisse, C., Mann, I., McCauley, P., Opitom, C., Ramanjooloo, Y., Raymond, J., Snodgrass, C., Steckloff, J. and Weissman, P. (2017) The Science of Near-Sun Comets. Space Science Reviews, unpublished.

Submit or recommend next manuscript to SCIRP and we will provide best service for you:

Accepting pre-submission inquiries through Email, Facebook, LinkedIn, Twitter, etc. A wide selection of journals (inclusive of 9 subjects, more than 200 journals)

Providing 24-hour high-quality service

User-friendly online submission system

Fair and swift peer-review system

Efficient typesetting and proofreading procedure

Display of the result of downloads and visits, as well as the number of cited articles Maximum dissemination of your research work

Submit your manuscript at: http://papersubmission.scirp.org/

Or contact jamp@scirp.org 